\title{
Erratum
}

\section{Congruence schemes}

\author{
[Int. J. Math., Vol. 24, No. 2 (2013) 1350009 (46 pages)] \\ Anton Deitmar \\ Mathematisches Institut \\ Auf der Morgenstelle 10 \\ 72076 Tübingen, Germany \\ deitmar@uni-tuebingen.de
}

Published 3 July 2015

Problem. As was pointed out to me by Peiyi Cui, the proof of Theorem 3.12 of that paper is flawed. A sesquiad homomorphism of the given type cannot exist, since the element $(-1)$ is contained in the sesquiad $A$ and as we have $(-1)^{2}=1$ as well as $1+(-1)=0$, the element $(-1)$ can neither be mapped to 0 nor to 1 , hence a morphism $\phi: A \rightarrow \mathbb{F}_{1}$ does not exist.

Solution. We give a different construction of a Tits model for $\mathrm{GL}_{n}$ by starting off from a different description of the coordinate ring $R$ of $\mathrm{GL}_{n}$ as follows:

$$
R=\mathbb{Z}\left[X_{i j}, Y_{i j}\right] / I,
$$

where $1 \leq i, j \leq n$, so we attach $2 n^{2}$ variables to $\mathbb{Z}$ and $I$ is the ideal generated by all elements of the form

$$
\sum_{k=1}^{n} X_{i k} Y_{k j}-\delta_{i j}
$$

where $\delta_{i j}$ is the Kronecker delta. Let $A \subset R$ be the multiplicative submonoid generated by all $X_{i j}$, all $Y_{i j}$ and all elements of the form

$$
X_{i 1}+X_{i 2}+\cdots+X_{i n}
$$

for $1 \leq i \leq n$. Let $\phi: A \rightarrow \mathbb{F}_{1}$ be a sesquiad morphism. Write $\phi\left(X_{i j}\right)=x_{i j}$ and $\phi\left(Y_{i j}\right)=y_{i j}$. Since the sum $X_{i 1}+\cdots+X_{i n}$ is in $A$, but $\mathbb{F}_{1}$ has trivial addition, at most one of the elements $x_{i 1}, \ldots, x_{i n}$ can be nonzero. We prolong $\phi$ by $\mathbb{F}_{1} \rightarrow \mathbb{Z} \rightarrow \mathbb{Q}$ to a sesquiad homomorphism $\eta: A \rightarrow \mathbb{Q}$ which therefore maps $X$ to an invertible 


\section{A. Deitmar}

matrix which has all entries equal to 0 or 1 with at most one entry nonzero in each row, therefore it must be a permutation matrix. On the other hand, each permutation matrix defines a sesquiad morphism $\phi$ and so

$$
\operatorname{Hom}\left(A, \mathbb{F}_{1}\right) \cong \operatorname{Per}(n)=W_{G} .
$$

